\title{
Outstanding Response to Sorafenib in a Patient with Metastatic Gastrointestinal Stromal Tumour
}

\author{
Charlotte Brinch $^{a} \quad$ Marie Dehnfeld ${ }^{a}$ Estrid Hogdall ${ }^{b}$ \\ Tim Svenstrup Poulsen $^{b}$ Anders Toxvaerd $^{b}$ Gina Al-Farra ${ }^{c}$ \\ Magnus Bergenfeldt ${ }^{d}$ e Anders Krarup-Hansen ${ }^{a}$ \\ aDepartment of Oncology, Herlev and Gentofte Hospital, Copenhagen University Hospital, \\ Copenhagen, Denmark; ${ }^{b}$ Department of Pathology, Herlev and Gentofte Hospital, \\ Copenhagen University Hospital, Copenhagen, Denmark; 'Department of Radiology, \\ Herlev and Gentofte Hospital, Copenhagen University Hospital, Copenhagen, Denmark; \\ ${ }^{\mathrm{d} D e p a r t m e n t}$ of Surgery, Skaane University Hospital, Lund, Sweden; ${ }^{\text {eDepartment of }}$ \\ Surgical Gastroenterology, Herlev and Gentofte Hospital, Copenhagen University Hospital, \\ Copenhagen, Denmark
}

\section{Keywords}

Gastrointestinal stromal tumour - Sorafenib - PDGFRA mutation - Multidisciplinary approach

\begin{abstract}
Gastrointestinal stromal tumour (GIST) is the most common sarcoma and can be seen in any part of the gastrointestinal tract. The effect of tyrosine kinase inhibitors varies with mutation status in receptor tyrosine kinase KIT and in platelet-derived growth factor receptor A (PDG$F R A$ ). This case presents a 61-year-old man, diagnosed with an 11-cm GIST located at the stomach with a high risk of recurrence. The patient showed intolerance to imatinib shortly after introduction and subsequently progressed on sunitinib and nilotinib. The patient started fourth-line treatment with sorafenib with an impressive response to a point at which metastases intra-abdominally and in the liver could be resected. After surgery, sorafenib was restarted. Due to toxicity, sorafenib dose was reduced over time. The dose was insufficient to control the disease since a new recurrence was detected. Mutation analyses revealed a GIST harbouring a deletion of codon p.1843_D846del, located at PDGFRA exon 18, right next to the codon D842 where mutations are known leading to imatinib resistance. In this case, the GIST was highly sensitive to sorafenib, and the response was dose related. It is mandatory to perform mutation analyses on primary tumour and at recurrence in the decision-making of the correct treatment for the patient. In March 2021, the patient had been in treatment with
\end{abstract}


sorafenib for 12.5 years and was still without signs of recurrence. A multidisciplinary approach was essential for the long-term survival of the patient in this case.

\section{Introduction}

Gastrointestinal stromal tumour (GIST) is the most frequent sarcoma with an incidence of 12-14 cases per million in Denmark [1,2]. GIST predominantly originates from the stomach (40-60\%), but can be seen in every part of the gastrointestinal tract [3]. Gene aberrations in receptor tyrosine kinase KIT $(c-K I T)(\sim 80 \%)$ and in platelet-derived growth factor receptor A (PDGFRA) (5-10\%) are commonly seen in GIST. The remaining 10-15\% of GISTs lack mutations in these genes and are called KIT/PDGFRA wild type [4,5]. The risk of tumour progression is evaluated by the Armed Forces Institute of Pathology (AFIP) criteria based on mitotic count, tumour size, and site [3].

The effect of tyrosine kinase inhibitors (TKIs) varies with mutation status in $c$-KIT and PDGFRA. Mutation analysis is therefore important in the diagnostics and has a prognostic value [2]. In the treatment of GIST, there are currently by June 20214 approved oncological treatments: imatinib, sunitinib, regorafenib, and avapritinib. Other TKIs such as sorafenib have also showed effect in the treatment of GIST. The following case report describes a patient course with outstanding response and survival on treatment of GIST with sorafenib.

\section{Case Report}

A 61-year-old man, previously radically resected for a renal cell carcinoma, was diagnosed with an 11-cm tumour located at the stomach 15 years ago (November 2005) (shown in Fig. 1.1; Table 1). This gastric tumour was primarily resected with free margins. Histopathology reported an epithelioid GIST, immunohistochemically positive for CD117 and CD34. A mitotic count of 20 per 50 high-power fields under lineated high risk of progression was found according to the AFIP criteria [3].

In April 2006, a recurrence in the lymph nodes in the lower pelvic and in the inguinal was discovered on imaging. First-line treatment with imatinib $400 \mathrm{mg}$ orally per day was initiated. The disease progressed in May 2006, and thereby the dose of imatinib was increased to 400 mg orally twice daily. A week later, the patient developed epidermolysis. The treatment was stopped in July 2006 after 3 months of treatment due to imatinib intolerance. Due to the skin reaction, prednisolone $50 \mathrm{mg}$ orally was administered. The following PET-CT showed regression of the disease, and therefore it was concluded that the presumed recurrence most likely had been a reactive change in the lymph nodes since it disappeared while on treatment with prednisolone.

Sixteen months after stopping imatinib, PET-CT revealed a recurrence with 3 intraabdominal tumours of $4.5 \mathrm{~cm}, 3.1 \mathrm{~cm}$ close to the liver and $2.6 \mathrm{~cm}$ close to the gall bladder (shown in Fig. 1.2), and it was verified by biopsy. Treatment with imatinib $200 \mathrm{mg}$ orally once daily was reintroduced. Again, the patient developed a severe skin reaction after a few days of treatment. Second-line treatment with continuous sunitinib $25 \mathrm{mg}$ orally once daily was initiated covered by 3-week treatment with prednisolone $25 \mathrm{mg}$ daily. The dose was then increased to $37.5 \mathrm{mg}$ sunitinib orally daily.

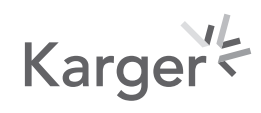




\section{Case Reports in Oncology}
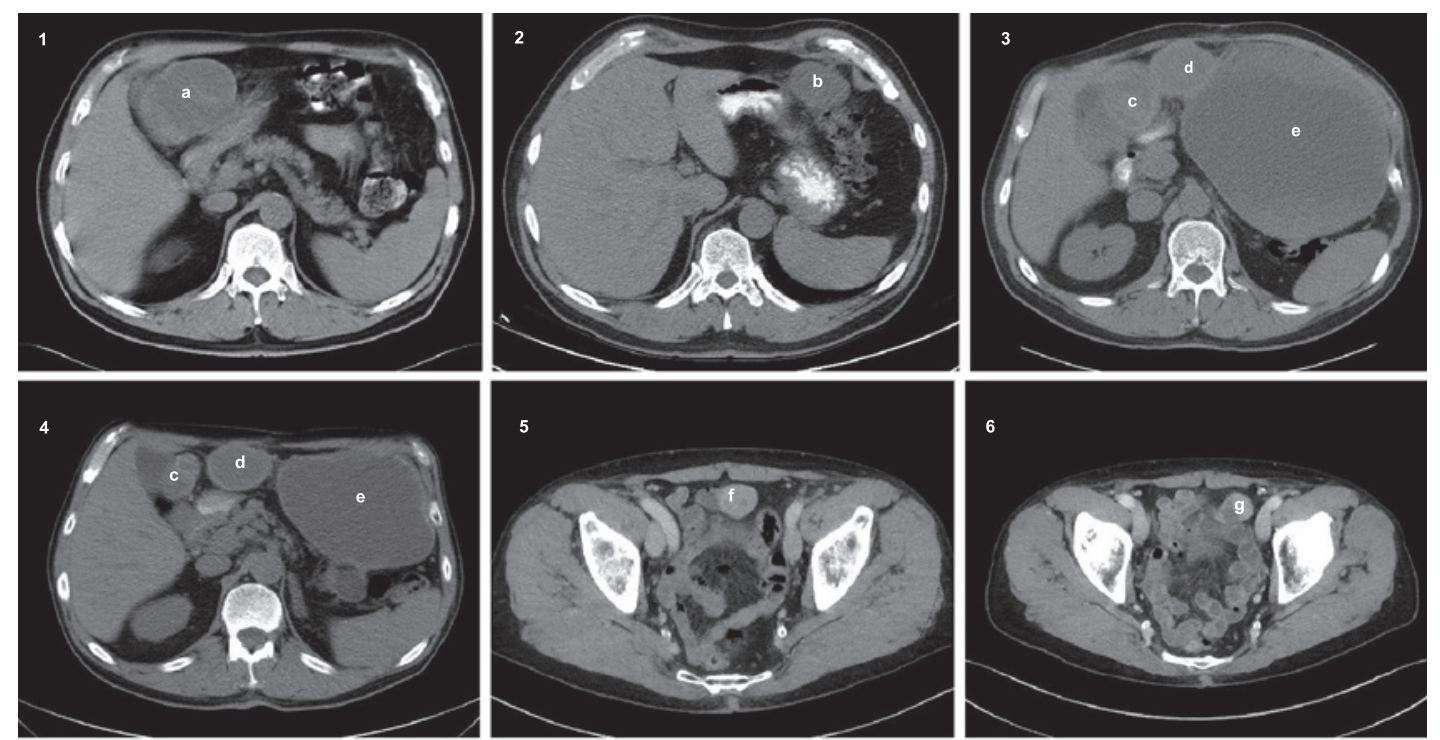

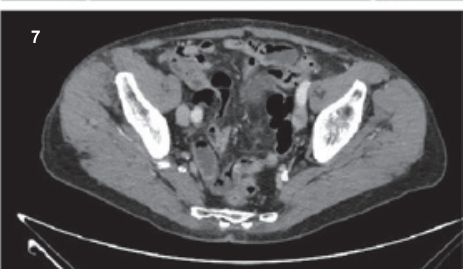

Fig. 1. 1: November 2005, primary tumour (a). 2: November 2007, image of recurrence showing 1 of 3 tumours (b). 3: August 2008, progression of disease (c-e [cystic with a peripheral solid component]). Baseline image when starting treatment with sorafenib. 4: August 2009, partial regression of tumour (c, d). The tumour (e) was not evaluated due to the cystic structure. 5: September 2017, progression of disease (f) after resection in September 2009. 6: January 2018, 15\% reduction in tumour size (g) after increased dose of sorafenib. Tumour was resected in February 2018. 7. March 2021, no signs of recurrence.

After 3 months of treatment with sunitinib, the disease progressed on PET-CT scan in 2 tumours intra-abdominally. Third-line treatment with nilotinib was initiated, $400 \mathrm{mg}$ orally twice daily. After 1 month of treatment, a new CT scan of the abdomen revealed progression of one of the existing tumours in the abdomen and in the left liver lobe with 2 new tumours. In lack of other treatment options, imatinib $200 \mathrm{mg}$ orally once daily was reintroduced with steroid coverage with prednisolone $100 \mathrm{mg}$ orally once daily. Unfortunately, the patient developed a urticarial rash, and the treatment was interrupted. Again, due to lack of treatment options, a second go with nilotinib was tried.

In June 2008, the disease progressed, but the treatment continued until the next CT scan. In August 2008, a cystic tumour process with a peripheral solid component in the pelvis was drained for 3 L of bloody fluid. A CT scan confirmed further progression in August 2008 (shown in Fig. 1.3). At that time, treatment with sorafenib became available and was administered as $400 \mathrm{mg}$ orally twice daily. After 1 month, the dose was reduced to $400+200 \mathrm{mg}$ orally due to toxicity. On treatment with sorafenib, the patient had partial regression with measurable targets according to Response Evaluation Criteria in Solid Tumours (RECIST) 1.1 regressing from $13.7 \mathrm{~cm}$ to $9.5 \mathrm{~cm}$ during a 1-year period (shown in Fig. 1.4) [6]. In September 2009 , the patient underwent surgery including cholecystectomy, partial liver resection, and resection of peritoneal disease. No residual tumour was left behind. Postoperatively, sorafenib was restarted at $200 \mathrm{mg}$ orally twice daily. 
Table 1. Timeframe of treatment and events

\begin{tabular}{|c|c|}
\hline Time & Event \\
\hline November 2005 & CT scan of primary tumour and surgery \\
\hline April 2006 & $\begin{array}{l}\text { Recurrence on CT imaging in the lymph nodes in the lower pelvic and in } \\
\text { inguinal }\end{array}$ \\
\hline April 2006 & First-line imatinib $400 \mathrm{mg} \times 1$ orally daily \\
\hline May 2006 & Progression* on CT imaging \\
\hline July 2006 & Imatinib dose escalation to $400 \mathrm{mg} \times 2$ orally daily \\
\hline July 2006 & Imatinib stopped due to allergic reaction \\
\hline August 2006 & $\begin{array}{l}\text { Imaging with no signs of recurrent GIST. In conclusion, the presumed tumour } \\
\text { recurrence on CT imaging was most likely a reactive change }\end{array}$ \\
\hline
\end{tabular}

November 2007

PET-CT shows 3 intra-abdominal tumours

Figure

November 2007

Reintroduction of imatinib $200 \mathrm{mg} \times 1$ orally daily. Stopped due to allergic reaction

November 2007

Second-line sunitinib $25 \mathrm{mg} \times 1$ increased to $37.5 \mathrm{mg} \times 1$ orally daily

February 2008

Progression* of disease

February 2008

Third-line nilotinib $400 \mathrm{mg} \times 2$ orally daily

March 2008

Progression* of disease and no treatment options

March 2008

Reintroduction of imatinib $200 \mathrm{mg} \times 1$ orally daily during prednisolone coverage. Stopped again due to allergic reaction

April 2008

Reintroduction of nilotinib $200 \mathrm{mg} \times 2$ orally daily, increasing the dose gradually to nilotinib $400 \mathrm{mg} \times 2$ orally daily

June 2008

Progression* of disease

August 2008

Baseline CT imaging before starting treatment with sorafenib

Fig. 1.1

September 2008

Fourth-line sorafenib $400 \mathrm{mg} \times 2$ orally daily

August 2009

Partial regression* of the disease on CT scan

Fig. 1.2

September 2009

Complete resection of multiple GIST metastases intra-abdominally. Resumes sorafenib $200 \mathrm{mg} \times 2$ orally daily postoperative

September 2012 Non-ST segment elevation myocardial infarct treated with a coronary artery bypass graft

May 2013

The dose of sorafenib was reduced further due to toxicity and was at this time $200 \times 1 \mathrm{mg}$ orally daily

September 2015 Non-ST segment elevation myocardial infarct treated with a percutaneous coronary intervention

October 2016 Diagnosed with proteinuria which was treatment related

September 2017 Progression* of disease. CT scan shows a tumour in relation to the terminal ileum

Fig. 1.5

October 2017 Increased dose of sorafenib to $400 \mathrm{mg}$ orally once daily

January 2018 CT scan shows 15\% reduction of tumour size

Fig. 1.6

February 2018 Macroradical surgery of GIST recurrence. Continued treatment with sorafenib $400 \mathrm{mg} \times 1$ orally daily

March 2021

Still no signs of GIST recurrence

Fig. 1.7

GIST, gastrointestinal stromal tumour.

*According to RECIST 1.1 [7]. 
Over the next years, full dose of sorafenib was not tolerated due to side effects, especially diarrhoea. In May 2013, the dosage was 200 mg orally per day.

Even though the dose of sorafenib was reduced, the patient had 2 non-ST segment elevation myocardial infarcts. Therefore, it was necessary to perform a coronary artery bypass graft in 2012 and a percutaneous coronary intervention in 2015. In addition, an investigation was carried out due to proteinuria which was concluded to be caused by a mild chronic kidney insufficiency. The cardiac and kidney affection was treatment related.

In April 2014, a mutation analysis was performed on archived material from the primary tumour from 2005 which revealed a deletion in exon 18 of PDGFRA involving c.2527. 2538delATCATGCATGAT, p.Ile843_Asp846del (p.I843_D846del), and wild-type c-KIT. At primary diagnosis in 2005 , mutation analysis was not part of daily practice.

In September 2017, a recurrence of GIST in relation to the ileum was discovered on CT imaging which retrospectively could be detected already in August 2016, and biopsy was carried out (shown in Fig. 1.5). The disease had then been stable, without recurrence, in 95 months during adjuvant treatment with sorafenib after resection of recurrence. Histopathology confirmed the diagnosis of GIST and an unchanged mutation status. Sorafenib was increased to $400 \mathrm{mg}$ orally once daily. Three months after dose escalation, image evaluation showed a 15\% reduction in tumour size (shown in Fig. 1.6). Since the patient was in performance status 0, resection of the tumour of $2.7 \mathrm{~cm}$ was performed in February 2018. Resection was complete with free margins.

After tumour resection, sorafenib $400 \mathrm{mg}$ orally once daily was reintroduced, and the patient is still in treatment with the same dose of sorafenib in March 2021. The patient had acceptable side effects such as diarrhoea and hypertension and is still without signs of recurrence (shown in Fig. 1.7).

\section{Discussion}

This case presents a patient with a GIST harbouring a deletion, p.I843_D846del, in PDGFRA exon 18 with an outstanding response to sorafenib. Gene aberrations located at PDGFRA are present in 5-10\% of GISTs [4,5]. Of all PDGFRA aberrations, substitution at codon D842 located at exon 18 constitutes 63\% while the deletions p.D842_H845 (DIMH842-845) and p.I843_D846 (IMH843-846) account for 15\% [7]. GISTs with the substitution at codon D842 in PDGFRA exon 18 are imatinib resistant [8] while GISTs with the deletions DIMH842-845/IMH843-846 are not [7]. The patient in this case report harboured a deletion of IMH843-846, leaving the aspartate intact at codon 842 (D842) in PDGFRA exon 18.

Imatinib, the first-line treatment for patients with GIST, inhibits few kinases including protein KIT and PDGFR $\alpha$ [9]. The patient in this case report showed intolerance to imatinib shortly after introduction, resulting in treatment effect not being evaluable. Subsequently, the patient progressed on sunitinib and nilotinib. The patient started fourth-line treatment with sorafenib with an impressive response to a point at which metastases intra-abdominally and in the liver could be resected. After surgery, sorafenib was restarted. Due to toxicity, diarrhoea, and hypertension, sorafenib dose was reduced over time. The dose was too low to control the disease since a new recurrence was detected in September 2017. In this case, a strong dose-response relation was observed.

Sorafenib, a multikinase inhibitor, is not approved for treatment of GIST but was the only available treatment option in 2013 when the patient in this case started this treatment. A phase II study from 2012 investigating sorafenib as the third-line treatment in patients with advanced GIST reported a median PFS of 4.9 months [10]. The median PFS was similar, 5.2 months, in another phase II study where the majority of the patients received sorafenib as

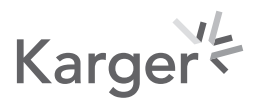


third-line treatment (84.2\%) [11]. A retrospective study has also investigated sorafenib in patients being resistant or intolerant to at least imatinib and sunitinib [12]. This study showed a median PFS of 6.4 months. Later, regorafenib has been approved as third-line treatment for patients with GIST. Sorafenib and regorafenib are similar but have different biochemical mechanisms of action against protein VEGFR-2, PDGFR $\beta$, FGFR1, KIT, and TIE-2 [13]. In 2013, a phase III study investigating regorafenib versus placebo in a third-line setting in patients with advanced GIST showed a median PFS of 4.8 months which was similar to sorafenib [14]. No published data are available regarding the effect of sorafenib or regorafenib in patients with a deletion of codon p.I843_D846del, located at PDGFRA exon 18.

\section{Conclusion}

The patient in this case had a GIST with a deletion of codon p.I843_D846del, located at PDGFRA exon 18 which was highly sensitive to sorafenib, and the response was dose related. GISTs with a mutation at codon D842, in PDGFRA exon 18, are known to be resistant to imatinib and other TKIs. Biopsy is important to secure the diagnosis and mutation status before treatment start in primary tumour and at recurrence. A multidisciplinary approach was essential for the long-term survival of the patient in this case.

\section{Acknowledgment}

Special thanks are due to the patient for allowing us to write this case report.

\section{Statement of Ethics}

The study is exempt from ethics committee approval since written informed consent was obtained from the patient for publication of this case report and any accompanying images.

\section{Conflict of Interest Statement}

The authors have no conflicts of interest to declare.

\section{Funding Sources}

The authors received no financial support for the authorship.

\section{Author Contributions}

Charlotte Brinch, Marie Dehnfeld, and Anders Krarup-Hansen drafted the manuscript; Anders Krarup-Hansen provided the clinical information; Estrid Hogdall and Tim Svenstrup Poulsen provided the molecular biological information; Anders Toxvard provided the pathological information; Gina Al-Farra evaluated treatment response on images and chose images for publication; Magnus Bergenfeldt provided surgical information; all authors participated in manuscript revision.

\section{Karger'}




\section{Data Availability Statement}

Data are available on request.

\section{References}

1 Aggerholm-Pedersen N, Rasmussen P, Dybdahl H, Rossen P, Nielsen OS, Safwat A. Serum natrium determines outcome of treatment of advanced GIST with imatinib: A Retrospective Study of 80 Patients from a Single Institution. ISRN Oncol. 2011;2011:523915.

2 Zhao X, Yue C. Gastrointestinal stromal tumor. J Gastrointest Oncol. 2012 Sep;3(3):189-208.

3 Miettinen M, Lasota J. Gastrointestinal stromal tumors: pathology and prognosis at different sites. Semin Diagn Pathol. 2006;23(2):70-83.

4 Corless CL, Fletcher JA, Heinrich MC. Biology of gastrointestinal stromal tumors. J Clin Oncol. 2004 Sep 15; 22(18):3813-25.

5 National Comprehensive Cancer Network. Gastrointestinal stromal tumors (GISTs) version 1.2021. 2020.

6 Eisenhauer EA, Therasse P, Bogaerts J, Schwartz LH, Sargent D, Ford R, et al. New response evaluation criteria in solid tumours: revised RECIST guideline (version 1.1). Eur J Cancer. 2009 Jan;45(2):228-47.

7 Corless CL, Schroeder A, Griffith D, Town A, McGreevey L, Harrell P, et al. PDGFRA mutations in gastrointestinal stromal tumors: frequency, spectrum and in vitro sensitivity to imatinib. J Clin Oncol. 2005 Aug 10;23(23): 5357-64.

8 Heinrich MC, Corless CL, Demetri GD, Blanke CD, von Mehren M, Joensuu H, et al. Kinase mutations and imatinib response in patients with metastatic gastrointestinal stromal tumor. J Clin Oncol. 2003 Dec 1;21(23): 4342-9.

9 Bauer S, Joensuu H. Emerging agents for the treatment of advanced, imatinib-resistant gastrointestinal stromal tumors: current status and future directions. Drugs. 2015 Aug;75(12):1323-34.

10 Park SH, Ryu MH, Ryoo BY, Im SA, Kwon HC, Lee SS, et al. Sorafenib in patients with metastatic gastrointestinal stromal tumors who failed two or more prior tyrosine kinase inhibitors: a phase II study of Korean gastrointestinal stromal tumors study group. Invest New Drugs. 2012 Dec;30(6):2377-83.

11 Kindler HL, Campbell NP, Wroblewski K, Maki RG, D’Amo DR. Sorafenib (SOR) in patients (pts) with imatinib (IM) and sunitinib (SU)-resistant (RES) gastrointestinal stromal tumors (GIST): Final results of a university of Chicago phase ii consortium trial. J Clin Oncol. 2011;29(15):10009. Chow.

12 Montemurro M, Gelderblom H, Bitz U, Schütte J, Blay JY, Joensuu H, et al. Sorafenib as third- or fourth-line treatment of advanced gastrointestinal stromal tumour and pretreatment including both imatinib and sunitinib, and nilotinib: a retrospective analysis. Eur J Cancer. 2013 Mar;49(5):1027-31.

13 Rutkowski P, Jagielska B, Andrzejuk J, Bylina E, Lugowska I, Switaj T, et al. The analysis of the long-term outcomes of sorafenib therapy in routine practice in imatinib and sunitinib resistant gastrointestinal stromal tumors (GIST). Contemp Oncol. 2017;21(4):285-9.

14 Demetri GD, Reichardt P, Kang YK, Blay JY, Rutkowski P, Gelderblom H, et al. Efficacy and safety of regorafenib for advanced gastrointestinal stromal tumours after failure of imatinib and sunitinib (GRID): an international, multicentre, randomised, placebo-controlled, phase 3 trial. Lancet. 2013 Jan 26;381(9863):295-302.

\section{Karger ${ }^{\prime}=$}

